\title{
Antonio Borghesi: il ricordo dei suoi allievi
}

Ilenia Confente - Barbara Gaudenzi - Ivan Russo - Paola Signori

Il prof. Antonio Borghesi è mancato all'affetto dei suoi parenti, amici e colleghi la sera del 23 febbraio 2017 all'età di 67 anni. Con forza e determinazione ha combattuto, durante gli ultimi tre anni, contro un male che mette a dura prova corpo e spirito, ma che lui ha fronteggiato con grande coraggio. Nell'ultimo anno ha tenacemente continuato i suoi amati studi, sempre in Università, nel vivo confronto con i colleghi e negli impegni istituzionali e accademici. Una vita forte e intensa, guidata da sogni divenuti spesso progetti ambiziosi. Resta ai suoi allievi ora il doloroso privilegio di ricordarlo, per raccontare il Professore di successo e per svelare l'Uomo che non tutti possono dire di aver conosciuto davvero.

$\hat{E}$ davvero difficile riassumere in poche pagine le gesta di chi, come Antonio Borghesi, ha sempre vissuto intensamente. Il profilo di un uomo come quello del prof. Borghesi si potrebbe rappresentare come "un manager prestato all'università" e allo stesso tempo come "un professore prestato alla politica", con una nota in comune: in tutti i ruoli che ha svolto è stato un leader, sempre pronto a battersi come un guerriero per le cose in cui credeva. Era una persona decisa, una guida con idee chiare e intuizioni originali, spesso di poche parole ma nette, dirette, pungenti fino ad essere abrasive per difendere le sue idee e oneste convinzioni.

$\mathrm{Fu}$ un professore poliedrico, sistemico nel suo ragionamento, rigoroso, autorevole e severo con i collaboratori, ma per loro è stato soprattutto un grande Maestro.

Fin dai suoi inizi, l'avventura universitaria di Antonio Borghesi si dimostrò particolare. Dopo la laurea (in Economia e Commercio, conseguita nel 1972 presso l'Università di Padova) il suo pragmatismo lo portò in azienda, dove giovanissimo già ricoprì ruoli di responsabilità. Le sue doti dirigenziali risultarono già evidenti, ma lui, avendo altre ambizioni, dopo pochi anni colse l'opportunità di iniziare a collaborare come Ricercatore e poi Professore incaricato di Economia e Finanza delle Imprese di Assicurazione presso l'Università di Padova, in sede veronese (l'ateneo di Verona diverrà sede autonoma dal 1982). Da qui ebbe avvio la sua vita universitaria, che si delineò poi più orientata alla Tecnica Industriale e Commerciale. Divenne così uno degli allievi del Prof. Panati che lui spesso richiamava come il suo Padre accademico.

Antonio Borghesi amava raccontare la sua storia accademica ad ogni nuovo allievo per ricordare sempre le radici della disciplina da cui proveniamo e per tramandarle nei nostri scritti, con spirito innovativo ma sempre con un profondo rispetto del passato. Quelle che lui considerava le basi della nostra area scientifica e di ricerca erano racchiuse in alcuni tomi scritti da quei maestri che anche lui spesso citava; libri e pubblicazioni che lui conservava nel suo ufficio e poi prestava ai vari allievi che dovevano preparare i loro esami di dottorato. 
sinergie Vol. 35, N. 102, 2017

I suoi studi sul turismo, sui distretti industriali, sui sistemi, sulla logistica, sul rapporto tra logistica e marketing e in generale sui temi del supply chain management e del risk management, hanno lasciato più di una impronta nei filoni di ricerca dell'Accademia Italiana, anche su questa rivista che ospita il Suo ricordo e con la quale collaborò attivamente.

Tra le sue prime pubblicazioni scientifiche Borghesi scrisse un'interessante e ancora attuale monografia su "Lorario flessibile" (1977), trattando ragioni e cause sociali, ambientali, psicologiche, biologiche e aziendali dell'importanza dell'orario flessibile per i lavoratori, offrendo minuziosamente dettagli operativi sui relativi piani di preparazione e conseguenze dell'introduzione di tali modelli nelle imprese. Nell'introduzione al suo lavoro Antonio scrisse "La ricerca della libertà da parte delluomo non ha mai conosciuto soste nel corso dei secoli. (..) Comprendere la vera essenza dellorario flessibile nel suo contenuto più estensivo, significherà dare un tangibile contributo alla secolare lotta dell'uomo per recuperare sé stesso e quello che, in termini di autonomia, la storia e lo sviluppo tecnologico gli hanno tolto. Non può essere che questo lauspicio di chi crede nel progresso civile e sociale dei popoli" (1977, p. 9). In molti suoi scritti e scelte successive emergerà il desiderio di lasciare un contributo tangibile al fine di creare valore per la società nel suo complesso.

Secondo il suo approccio scientifico (che oggi denomineremmo "theory building") Borghesi lamentava una "frattura tra teoria e prassi", criticando il dibattito del tempo "più incentrato sui modelli organizzativi e meno alla discussione attorno alle teorie ad essi sovrastanti" (1984, p. 137). Per questo, nel suo lavoro su "Sistemi e organizzazioni economiche" (1984) tentò di delineare una serie di leggi della teoria generale dei sistemi. Oltre a definire alcune caratteristiche identificative del concetto di sistema, Borghesi discute di leggi della teoria generale dei sistemi aperti (Entropia negativa o Neghentropia; Equifinalità; Differenziazione; Integrazione; Competizione), con relativi corollari e conseguenze sulle organizzazioni economiche. Riteneva infatti che "una costruzione logica si qualifichi come teoria non solo quando scopre nuove leggi, ma anche quando trasforma principi (di per sé già esistenti) in leggi” (1984, p. 138).

Antonio Borghesi divenne Professore Ordinario in Finanza Aziendale nel 1990, a soli 41 anni, vincendo la cattedra all'Università della Calabria. La parentesi calabrese, pur se istituzionalmente breve, rimase poi sempre attiva e spesso richiamata nei suoi racconti come un periodo ricco di opportunità e di relazioni. In quegli anni, anche con i suoi primi allievi Sonia Ferrari e Alfio Cariola, si dedicò al marketing turistico pubblicando una curatela su "Il marketing delle località turistiche: aspetti metodologici e ricerche empiriche" (1994) e appena più tardi il saggio "Dal marketing turistico al marketing territoriale" (1997). Nel sottolineare l'importanza del legame tra territorio e turismo, Borghesi vuole far emergere quanto " $i l$ fenomeno turistico sia forse una delle attività umane che più di ogni altra crea ricadute nei più disparati settori delleconomia" (1994, p. 24). Nelle sue analisi si delineano integrazioni e disintegrazioni tipiche del settore, le fasi di lavorazione del processo terminale di produzione del servizio turistico (1994, pp. 19-24), aspetti critici, opportunità della filiera del turismo se osservata come sistema integrato. 
Nel 1993 tornò ad incardinarsi presso l'Università di Verona, come Professore Ordinario in Tecnica Industriale e Commerciale (oggi Economia e Gestione delle Imprese) e titolare della cattedra di Marketing. Furono anni in cui, grazie ai segnali captati nel suo ricco network di imprenditori e manager, si dedicò alla progettazione e attivazione di due corsi di perfezionamento di successo, tuttora tra i Corsi post-lauream più longevi dell'Università di Verona: il Corso di Perfezionamento in Risk Management (fondato nel 1994) e il Corso di Perfezionamento in Logistica Distributiva (nato nel 1995). Queste iniziative furono possibili grazie al sostegno e al contributo delle aziende, che fin da subito credettero nei suoi progetti.

Il prof. Borghesi per la sua intraprendenza era molto apprezzato nel mondo industriale e imprenditoriale veneto e fu per questo che gli venne proposto un incarico politico. Entrò così in politica, venendo eletto nel 1995 Presidente della Provincia di Verona. Mantenne comunque attivi tutti i suoi progetti scientifici e quindi anche l'attività di ricerca universitaria, come poi sempre farà durante tutte le sue scelte politiche. Tra i numerosi progetti, in particolare Borghesi era responsabile per l'Università di Verona dell'European Business Programme (EBP), un programma formativo internazionale in cui lo studente frequentava per metà del suo percorso universitario in Italia e l'altra metà in una sede estera del network, conseguendo due titoli di laurea equipollenti nelle due sedi di studio: un esempio di internazionalizzazione della didattica che agli inizi degli anni '90 era futuristica. Tra i primi Antonio Borghesi intuì la rilevanza di una apertura internazionale sia nella ricerca scientifica che nelle esperienze formative, spingendo tutti i suoi allievi a misurarsi in contesti internazionali, creando solidi legami con Università straniere.

In quegli anni Borghesi si impegnò anche in numerose ricerche sull'internazionalizzazione delle imprese e dei modelli distrettuali. In particolare si ricordano le sue concettualizzazioni di "distretto gemmato" e di "distretto simbiotico" (2002); il primo nasce quando alcune imprese distrettuali riproducono nei mercati internazionali modelli speculari a quelli di partenza, replicando modelli e routine organizzative sperimentali; il secondo è considerabile come la connessione tra distretto tradizionale e gemmato, quale uno dei possibili modelli di distretto allargato che potranno concretizzarsi ed evolvere nel tempo dato che inizialmente il distretto gemmato non è autonomo e necessita del supporto del distretto di partenza (2002, p. 32).

Aperto a studi e contesti internazionali, nel costante sforzo verso la ricerca dell'originalità e per lasciare un contributo tangibile, già a fine degli anni '90 il prof. Borghesi si intestardì per realizzare un suo sogno: trasformare il corso di Logistica (prima denominato Distributiva, poi Integrata) in un Master Universitario. La progettazione del Master richiese mesi, e con Paola Signori che nel frattempo Antonio aveva appena seguito come relatore nella sua tesi di dottorato sul supply chain management, decise di includere nel programma del master non solo competenze di logistica ma anche di supply chain management, appunto a quei tempi erano tematiche ancora poco note in Italia, i cui primi segnali d'interesse provenivano da organizzazioni europee e da aziende multinazionali che operavano negli Stati Uniti. Il piano formativo del LogiMaster (Master in Logistica - Supply
Ilenia Confente Barbara Gaudenzi Ivan Russo Paola Signori

Antonio Borghesi: il ricordo dei suoi allievi 
sinergie Vol. 35, N. 102, 2017

Chain Management) era davvero ambizioso e servivano risorse. Invece di affidarsi ad uno sponsor unico, il prof. Borghesi volle raccontare il suo progetto a tante imprese diverse e raccogliere numerosi partner. Decise dunque di organizzare un vero e proprio road show e in pochi mesi il progetto venne presentato presso 42 aziende, ottenendo il consenso da ben 18 per il primo anno. Il LogiMaster è sempre stato un suo orgoglio: ora già alla sedicesima edizione (nel 2017), ha diplomato oltre 330 allievi, e risulta il Master più longevo dell'Università di Verona.

Dal 2000 al 2002 Antonio Borghesi fu poi Direttore del Dipartimento di Studi Finanziari Industriali e Tecnologici dell'Università di Verona. Un piccolo aneddoto di quel periodo può far meglio capire come Borghesi amasse il suo lavoro: insediatosi come direttore, volendo portare unondata di vero cambiamento e semplificazione delle procedure con una netta riorganizzazione interna, si preoccupò per prima cosa di svolgere numerosi colloqui individuali col personale tecnico-amministrativo, per capire quali fossero i problemi e prospettare la fattibilità di alcune soluzioni. Le sue soluzioni erano fondate dalla condivisione, sempre basate su bisogni reali e discusse insieme a quelli che poi sarebbero stati i principali fautori del cambiamento. Allo stesso modo, con gli allievi, le scelte passavano attraverso accese discussioni ma con un metodo che prevedeva un ragionamento profondo e articolato. Spesso convocava riunioni di confronto scientifico aperto, denominate il "venerdì scientifico". Quando non sapeva "chiedeva" e si metteva in discussione senza ruoli, gerarchie o gerontocrazie affidandosi spesso ai più giovani per affrontare il cambiamento in ambito accademico.

Nacquero in queste sessioni le sue concettualizzazioni sul doppio posizionamento strategico, ben distinto dal posizionamento semplice, e ritenuto "indispensabile in tutti $i$ casi in cui gli influenzatori siano specificamente individuabili e il loro grado d'influenza sia altresì di alta o altissima intensità" (2002).

Borghesi andava spesso controcorrente, in molti ricorderanno i suoi accesi dibattiti sulletica d’azienda, su cui poi apparve un articolo nella rivista Sinergie (2005).

Antonio Borghesi era contemporaneamente un uomo di pensiero e un uomo del fare. Le sue analisi e riflessioni scientifiche nascevano e venivano poi spese in gruppi di lavoro nazionali e internazionali, con il preciso fine di realizzare i progetti di cui lui scriveva.

Tre sono i filoni principali che lo hanno accompagnato in tutta la sua vita accademica: il risk management, la logistica e il marketing.

Il risk management fu la sua prima area di ricerca, e già nel 1973 pubblicò il suo primo articolo sull'argomento. Successivamente divenne presidente del CESRAS-Centro per gli Studi sul Rischio e l'Assicurazione, membro accademico dell'A.R.I.A. (American Risk and Insurance Association), di RIMS (Risk and Insurance Management Society-USA), unico membro italiano del "Gruppo dei 22" riunitosi per la prima volta a Parigi nel gennaio del 1990 in seno allattuale FERMA (Federation of Risk Management Associations). Divenne, inoltre, presidente della Commissione Tecnica del Gruppo "Gestione del Rischio" in UNI (Ente Nazionale Italiano di Unificazione) e componente del Working Group on 
Risk Management in ambito ISO (International Standard Organisation)/ TMB che ha prodotto le norme ISO 31000 sulla gestione del rischio.

Pioneristica nel 1985 fu la sua prima sistemica pubblicazione sul tema "La gestione dei rischi di azienda. Economia e organizzazione. Teoria e pratica". Dai suoi studi sul risk management, ricavò le tecniche e le radici nella scienza del pericolo, ovvero della Chindinica Aziendale (1999), scrivendo di relativi assiomi, leggi, e deficienze sistemiche.

Fino ai più recenti contributi assieme alla sua allieva Barbara Gaudenzi che più si è dedicata al tema, in particolare nel 2006 con l'articolo "Managing risks in the supply chain using the AHP method" e successivamente con la monografia "Risk management. How to assess, transfer and communicate critical risks" nel 2013.

Dal 2000 fu membro accademico del Council of Supply Chain Management Professionals - USA, e si dedicò con passione ai temi del supply chain management e del servizio logistico, fu tra i primi ad intuire la relazione stretta tra il marketing e il servizio logistico pubblicando nel 2006 il libro "Marketing-Logistica" che era stato preceduto anche su questa rivista da altri contributi sul medesimo filone, tra cui la curatela "Logistica Integrata tra Teoria e Pratica: tendenze evolutive" nel 2001. In tale filone di ricerca Antonio Borghesi partì dal presupposto che tradizionalmente le attività di marketing e logistica sono gestite separatamente nella maggior parte delle imprese. Tuttavia, osservò come il servizio al cliente sia anche un comune denominatore delle due funzioni, ciò che risulta ben più evidente quando esse vengono integrate nel processo di gestione della catena di fornitura (Supply Chain Management), comprendendo anche i flussi di ritorno (2008). A tali processi viene assegnato l'obiettivo di "creazione di valore per il cliente" che si sostanzia in due elementi: il servizio di distribuzione fisica e il servizio (di marketing) al cliente. Da questo contributo e dall'evoluzione dei filoni di ricerca collegati in tali ambiti il prof. Borghesi si fece promotore di alcuni corsi incardinati nelle lauree magistrali dell'Università di Verona, unici nel loro genere in Italia all'interno della Facoltà di Economia, come ad esempio Customer Service \& Supply Chain Management o International Logistics \& Marketing Management ora impartiti dal suo allievo Ivan Russo.

Il Prof. Borghesi è sempre stato aperto ai giovani, affidandosi a loro per affrontare il cambiamento in ambito accademico, ad esempio nella scrittura degli articoli internazionali. Negli ultimi anni, anche insieme all'allieva Ilenia Confente, ha coltivato un dibattito e un aggiornamento scientifico sui temi di marketing e supply chain legati al contesto online e all'omni-canalità. Ne è conseguito uno studio (2015) sulla necessità per le imprese di reagire real-time ad un mercato che si avvale di più canali, attraverso l'acquisizione di capacità adattive e predittive che consentono alle imprese di raccogliere e analizzare i "big data" che provengono sia dal contesto offline che online.

Infine, tra i suoi ultimi interessi compare la "City Logistics", e le cause di fallimento di alcuni progetti italiani (2017).

Questi temi, così come quelli precedentemente illustrati, sono alcune testimonianze di come Antonio Borghesi abbia sempre condotto numerose ricerche con/per le imprese, caratterizzandosi con pragmatismo e teorizzazione scientifica, dinamismo appassionato e voglia di implementare nuovi modelli e approcci gestionali.
Ilenia Confente

Barbara Gaudenzi Ivan Russo

Paola Signori

Antonio Borghes:

ricordo dei suoi allievi 
sinergie Vol. 35, N. 102, 2017

I suoi allievi ricordano le molte visite aziendali, alla ricerca di un confronto continuo con le best practice. Borghesi lavorava senza sosta, con grande passione, e con il suo esempio spronava tutti i suoi collaboratori a dare il meglio soprattutto nel confronto scientifico, a viaggiare tra prestigiosi convegni che potessero offrire utili spunti alla crescita, verso un miglioramento continuo che avesse anche un significato. Ai giovani allievi chiedeva dedizione e umiltà, instaurando un rapporto franco e trasparente come quello del giocatore con il suo coach, spronandoli ad assumersi responsabilmente ruoli e autonomie, e nel contempo coltivando con ognuno di essi un rapporto unico e speciale, chiedendo a tutti subito di dargli del "tu". Grazie a lui hanno imparato a coltivare una mentalità che ha giovato nella maturazione professionale, e che oggi li accompagna per le sfide future.

Come leader, Antonio Borghesi ha sempre creduto nella squadra, l'ha difesa e coltivata anche grazie a iniziative informali, cariche di grande umanità e affetto. Un esempio è stato il viaggio a Münster del 2005: 1.000 $\mathrm{km}$ in auto, tutti insieme, per raggiungere la sede del World Marketing Congress organizzato dall'Academy of Marketing Science, il prestigioso convegno che nel 2007 Borghesi voleva portare a Verona. E così la frequenza a un convegno, motivata da motivi scientifici ed organizzativi, divenne una delle occasioni per vivere e assaporare il senso di una famiglia accademica. Tanti sono gli aneddoti che in vent'anni di lavoro i suoi allievi hanno collezionato, e che mantengono come preziosi ricordi del loro percorso accademico con lui, ma altrettante sono le idee innovative che Antonio ha lasciato in germe e che il suo gruppo sarà chiamato a realizzare in futuro.

Ora Antonio Borghesi lascia un vuoto incolmabile come maestro, come amico, e come uomo; tuttavia contemporaneamente ha lasciato uneredità unica, quale indelebile impronta e identità forte e strutturata allinterno del gruppo di ricerca da lui creato, in Supply Chain \& Marketing e che opera all'interno del Dipartimento di Economia Aziendale dell'Università di Verona.

Grazie Professore!

\section{Bibliografia}

BORGHESI A. (1977), Lorario flessibile, Franco Angeli Editore, Milano.

BORGHESI A. (1984), "Sistemi e Organizzazioni economiche", Economia e Politica Industriale, n. 41, pp. 137-161.

BORGHESI A. (1985), La gestione dei rischi di azienda, Cedam, TorinoPadova, 1985.

BORGHESI A. (1994), (a cura di) Il marketing delle località turistiche: aspetti metodologici e ricerche empiriche, Giappichelli Editore, Torino.

BORGHESI A. (1997), "Dal marketing turistico al marketing territoriale", Economia, società e istituzioni, n. 9, pp. 385-397. 
BORGHESI A. (2001), "Logistica Integrata tra teoria e pratica: tendenze evolutive”, Sinergie, n. 57, pp. I-V.

Ilenia Confente

Barbara Gaudenzi

Ivan Russo

Paola Signori BORGHESI A. (2002), "Il distretto simbiotico come nuovo modello di Antonio Borghesi: internazionalizzazione delle piccole e medie imprese distrettuali italiane", in Velo D., Majocchi A. (a cura di), L'internazionalizzazione delle piccole e medie imprese nell'Europa Centro Orientale, Giuffrè Editore, Milano.

BORGHESI A. (2006), Marketing - Logistica, Giuffrè Editore, Milano.

BORGHESI A. (2005), "Una riflessione provocatoria sulletica in azienda", Sinergie, n. 67, p. 67.

BORGHESI A. (2017), "City Logistics: Is Deregulation the Answer?", in: Bilgin M., Danis H., Demir E., Can U. (eds) Financial Environment and Business Development. Eurasian Studies in Business and Economics, vol 4. Springer, Cham, Svizzera.

BORGHESI A., SIGNORI P. (1999), "Chindinica aziendale: gli assiomi, le leggi, le deficienze sistemiche", in Baccarani C. (ed.), Scritti di Tecnica Economica sull'Organizzazione e la Finanza, Cedam, Padova, pp. 191-214.

BORGHESI A., SIGNORI P. (2002), "Il doppio posizionamento strategico. Considerazione teoriche ed evidenze empiriche", in II International Marketing Trends Conference Proceedings, ESCP, Paris.

GAUDENZI B., BORGHESI A. (2006), "Managing risks in the supply chain using the AHP method", The International Journal of Logistics Management, vol. 17, n. 1, pp. 114-136.

GAUDENZI B., BORGHESI A. (2013), Risk management. How to assess, transfer and communicate critical risks, Springer.

RUSSO I., BORGHESI A. (2008), "Il processo di gestione dei prodotti-ritorni”, Sinergie, n. 77, pp. 163-186.

RUSSO I., CONFENTE I., BORGHESI A. (2015), "Using big Data in the Supply Chain Context: Opportunities and Challenges", Proceedings of the $16^{\text {th }}$ European Conference on Knowledge Management ECKM 2015, pp. 649-656.

\section{Academic or professional position and contacts}

\section{Ilenia Confente}

Researcher of Management

University of Verona - Italy

e-mail: ilenia.confente@univr.it

\section{Barbara Gaudenzi}

Associate Professor of Management

University of Verona - Italy

e-mail: barbara.gaudenzi@univr.it

\section{Ivan Russo}

Associate Professor of Management

University of Verona - Italy

e-mail: ivan.russo@univr.it

\section{Paola Signori}

Associate Professor of Management

University of Verona - Italy

e-mail:paola.signori@univr.it

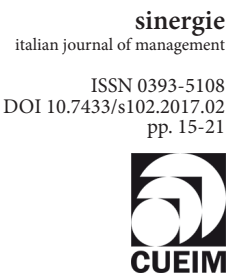

